\title{
The influence of perceived rotary motion on the recognition of rotated objects
}

\author{
PIERRE JOLICOEUR \\ University of Waterloo, Waterloo, Ontario, Canada \\ MICHAEL C. CORBALLIS \\ University of Auckland, Auckland, New Zeland \\ and \\ REBECCA LAWSON \\ University of Liverpool, Liverpool, England
}

\begin{abstract}
Subjects either named rotated objects or decided whether the objects would face left or right if they were upright. Response time in the left-right task was influenced by a rotation aftereffect or by the physical rotation of the object, which is consistent with the view that the objects were mentally rotated to the upright and that, depending on its direction, the perceived rotary motion of the object either speeded or slowed mental rotation. Perceived rotary motion did not influence naming time, which suggests that the identification of rotated objects does not involve mental rotation.
\end{abstract}

Many objects have a predominant orientation in the environment. The time required to identify such objects increases as they are rotated in the image plane from their usual upright orientation, up to about $120^{\circ}$ (Jolicoeur, 1985; Jolicoeur \& Milliken, 1989; Maki, 1986; Murray, 1995; Murray, Jolicoeur, McMullen, \& Ingleton, 1993; Shwartz, 1981). A number of researchers have proposed that mental rotation (see M. C. Corballis, 1982; Shepard \& Cooper, 1982), a continuous rotational transformation of a representation of the stimulus, could be responsible for the systematic effect of orientation on the recognition of rotated objects (see, e.g., Jolicoeur, 1985, 1990; Tarr \& Pinker, 1989).

Our aim in this article was to test whether the function relating response time (RT) to orientation when rotated objects are named is indeed due to mental rotation. We made use of an effect that has been found in experiments that are believed to induce mental rotation and that could represent a marker for mental rotation. It has previously been shown that the RT for deciding whether a rotated letter is normal or backward is influenced by the induction of a rotation aftereffect (M. C. Corballis \& McLaren, 1982). The effect is most marked at $120^{\circ}$ and $240^{\circ}$ clockwise from the upright. When the induced aftereffect is

This work was supported by a research grant from the Natural Sciences and Engineering Research Council of Canada, awarded to P. J., and by a Human Frontiers Science Program postdoctoral fellowship and Grant LT-558/94, awarded to R. L. We thank Marg Ingleton and Matt Williams for technical assistance and Johann Wagemans for useful comments and suggestions. Correspondence concerning this article should be addressed to P. Jolicoeur, Department of Psychology, University of Waterloo, Waterloo, ON, Canada N2L 3G1 (e-mail: pjolicoe@cgl.uwaterloo.ca). clockwise, RTs are longer for the $120^{\circ}$ than for the $240^{\circ}$ orientation; conversely, when the aftereffect is counterclockwise, RTs are longer for the $240^{\circ}$ than for the $120^{\circ}$ orientation.

A similar interaction is also found if the letter is in rotary motion during its presentation (Jolicoeur \& Cavanagh, 1992). Jolicoeur and Cavanagh suggested that mental rotation is speeded by perceived motion in the direction of rotation to the upright and slowed by perceived motion in the opposite direction. The interactions between direction of aftereffect and stimulus orientation (M. C. Corballis \& McLaren, 1982) and between direction of perceived motion and stimulus orientation (M. C. Corballis \& Blackman, 1990; P. M. Corballis \& M. C. Corballis, 1993; Jolicoeur \& Cavanagh, 1992) both appear to be markers for mental rotation.

In the present work, we made use of both kinds of interaction. Previous work in this area has used either alphanumeric characters or very simple stimuli, such as Lshaped patterns. We extended the previous work on these effects by showing that robust interactions can be found by using rotated drawings of objects in a left-right decision task that should induce mental rotation. Then we tested whether a similar interaction would be found in a naming task. In Experiment 1 we induced a rotary aftereffect that made the objects appear to rotate either clockwise or counterclockwise. In Experiment 2 we displayed the objects actually rotating either clockwise or counterclockwise. These two quite different methods produced clear-cut effects in the left-right task but none in the naming task. The results suggest that mental rotation was not responsible for the orientation effects observed when rotated objects were identified. 


\section{EXPERIMENT 1}

Two groups of subjects were tested. One group named rotated objects as quickly as possible. Before each trial, the subjects adapted to rotary motion (either clockwise or counterclockwise), such that, when the object to be named was displayed, it appeared to rotate in the opposite direction. The empirical question was whether the rotary aftereffect would interact with the orientation of the displayed object.

Another group of subjects judged whether the objects would face left or right if they were upright-a task thought to require mental rotation (Jolicoeur, 1985, 1988). We expected to find an interaction between the rotary aftereffect and object orientation in the left-right task, which would provide a conceptual replication of several previously reported findings (see, e.g., M. C. Corballis $\&$ McLaren, 1982) and evidence for the use of mental rotation. If a similar interaction was found in the naming task, the results would suggest that mental rotation was also used to identify a rotated object. The absence of this interaction would suggest that some other orientationsensitive mechanism was responsible for the effects of orientation on object identification.

\section{Method}

\section{Subjects}

There were 48 subjects, 36 women and 12 men, drawn from a 3rdyear undergraduate course in human neuroscience. Participation was voluntary.

\section{Stimuli}

A circle divided equally into 24 alternating black and white sectors was generated by computer on a VGA screen. The diameter was $17 \mathrm{~cm}$. It was displayed against a black background in order to give the appearance of a 12-spoked wheel and set to rotate at $120 \% \mathrm{sec}$ in either a clockwise or a counterclockwise direction. This stimulus induced a strong rotary motion aftereffect on a subsequent stationary stimulus.

Seventy-two drawings of common objects from Snodgrass and Vanderwart (1980) were digitized. When upright, 39 faced left and 33 faced right. The pictures were shown in white against a black back ground at one of three orientations: $0^{\circ}, 120^{\circ}$, or $240^{\circ}$ clockwise from upright. The drawings were scaled so that they were contained within the area of the rotating wheel and were therefore subject to the rotary aftereffect induced by the wheel.

\section{Design}

Each subject received two blocks of 36 trials and saw a different picture on each trial. There were 24 pictures ( 13 left-facing and 11 rightfacing when upright) in each of the three orientations $\left(0^{\circ}, 120^{\circ}\right.$, and $240^{\circ}$ ).

The subjects were divided into groups of three, such that, within each group, each picture was seen at $0^{\circ}$ by one subject, $120^{\circ}$ by another, and $240^{\circ}$ by the third. Each picture was therefore displayed equally often at each orientation. The order in which the pictures were presented was randomized for each group of 3 subjects.

\section{Procedure}

The subjects were divided into two groups of 24; the division was random, with the restriction that there were 18 women and 6 men in each group. One group named the pictures, and the other group decided whether each picture would face left or right if upright. Each group was then further subdivided so that, for half the subjects ( 9 women, 3 men), the wheel rotated clockwise and for the other half rotated counterclockwise.

Each subject was first given 15 practice trials, in which five objects not included in the experimental trials were presented at each of the three orientations. This was followed by the two blocks of experimental trials. Before the first practice trial and before the first trial of each block, the wheel rotated for $30 \mathrm{sec}$. It rotated for $3 \mathrm{sec}$ on subsequent trials. These conditions were sufficient to produce a strong motion aftereffect on each trial.

Immediately after the wheel disappeared, the picture appeared on the screen and remained there until the subject responded. Subjects in the naming group were asked to speak the name of each depicted object into a microphone. The actual name of the object then appeared on the screen, and subjects were asked to press the space bar if they had named the object correctly and the $\mathrm{N}$ key otherwise. They were permitted some license in naming the object; for example, bicycle or cycle were regarded as equivalent to bike. In the case of stimuli that belonged to classes, such as animals or birds, the subjects were required to name the actual species (e.g., cat, eagle) rather than give the generic name. Subjects in the left-right group were asked to indicate whether the objects would face left or right if upright by pressing the $Z$ or / key, respectively. In both groups, RTs were measured from the onset of the object to the subject's first response. The intertrial interval was $0.5 \mathrm{sec}$.

\section{Results}

Two kinds of analyses were performed: one based on data aggregated over subjects and one based on data aggregated over items. For the subjects analysis, the results are based on four groups of 12 subjects, with two betweensubjects factors - task (left-right vs. naming) and direction of aftereffect (clockwise vs. counterclockwise)and two within-subjects factors-orientation $\left(0^{\circ}, 120^{\circ}\right.$, and $240^{\circ}$ ) and face (left vs. right, coding whether the object faced left or right when upright). For the items analysis, all factors were treated as within-items.

\section{Subject Analyses}

Response times. The median RT for correct responses for each combination of orientation and face (which side the object faced) conditions was computed for each subject. The medians were then subjected to an analysis of variance (ANOVA) in which task (naming vs. left - right) and direction of aftereffect (clockwise vs. counterclockwise) were between-subjects factors. Although there were effects that were due to face, these effects did not interact with the direction of aftereffect, which is the main focus of the present work, so this factor will not be discussed further. Note that the direction of the aftereffect is opposite to the direction of wheel rotation. The means of the medians are shown in the top panel of Figure 1.

Naming times were longer $(1,082 \mathrm{msec})$ than leftright judgment times [ $814 \mathrm{msec} ; F(1,44)=46.90, M S_{\mathrm{e}}=$ $110,349, p<.0001]$. This result is not surprising, because the modes of response (vocal vs. manual) and the postperceptual requirements of the tasks (retrieving and producing a name vs. assigning left-right codes to manual responses) were quite different. This difference also replicates several earlier results (see, e.g., Jolicoeur, 1985, 1988). 


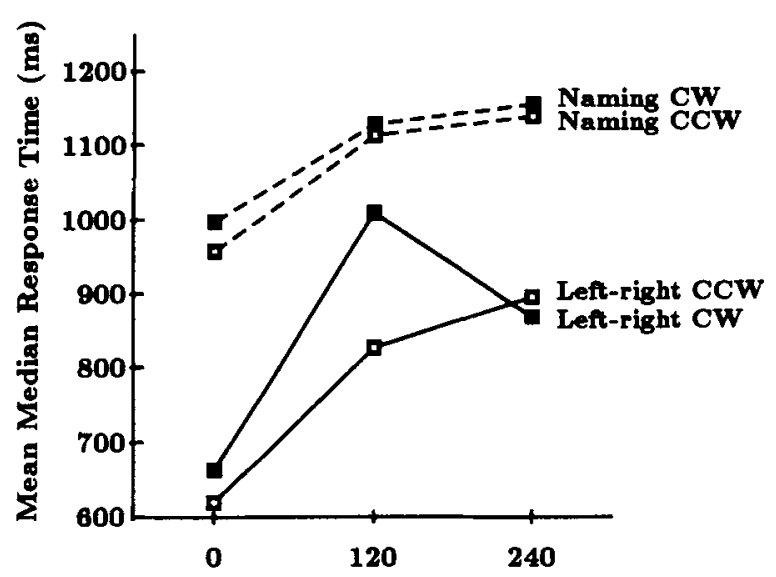

were highly reliable in both tasks $\left[F(2,44)=19.69, M S_{\mathrm{e}}=\right.$ $20,490.0, p<.0001]$ in the naming task; $F(2,44)=38.78$, $M S_{\mathrm{e}}=28,227.0, p<.0001$, in the left-right task].

The critical question was whether the direction of the aftereffect would influence the orientation function. The interaction between these variables approached significance $\left[F(2,88)=2.70, M S_{\mathrm{e}}=24,358.5, p<.074\right]$, as did the triple interaction between task, direction of aftereffect, and orientation $\left[F(2,88)=3.02, M S_{\mathrm{e}}=24,358.5, p<\right.$ $.055]$. The interaction between orientation and direction of aftereffect was clearly present for the left-right task but was negligible for the naming task. Since the direction of the aftereffect was expected to influence RT only at the $120^{\circ}$ and $240^{\circ}$ orientations (M. C. Corballis \& McLaren, 1982), the triple interaction was recomputed with the $0^{\circ}$ orientation removed, and it then proved to be significant $\left[F(1,44)=4.50, M S_{\mathrm{e}}=29,618.3, p<.04\right]$.

Separate ANOVAs were also carried out for each task. The interaction between the direction of the aftereffect and orientation was significant for the left-right task $[F(2,44)=$ 4.84, $M S_{\mathrm{e}}=28,227.0, p<.013$ ] but not for the naming task $\left[F(2,44)=0.12, M S_{\mathrm{e}}=20,490.0, p>.88\right]$, even when the results for $0^{\circ}$ were removed $[F(1,22)=0.0002$, $\left.M S_{\mathrm{e}}=21,622.7, p>.98\right]$. These analyses also provide evidence that the lack of influence of the aftereffect on the naming task was not due to very high error variance, because the $M S_{\mathrm{e}}$ estimate was smaller for the naming task than for the left-right task.

Accuracy. The proportion of correct responses in the

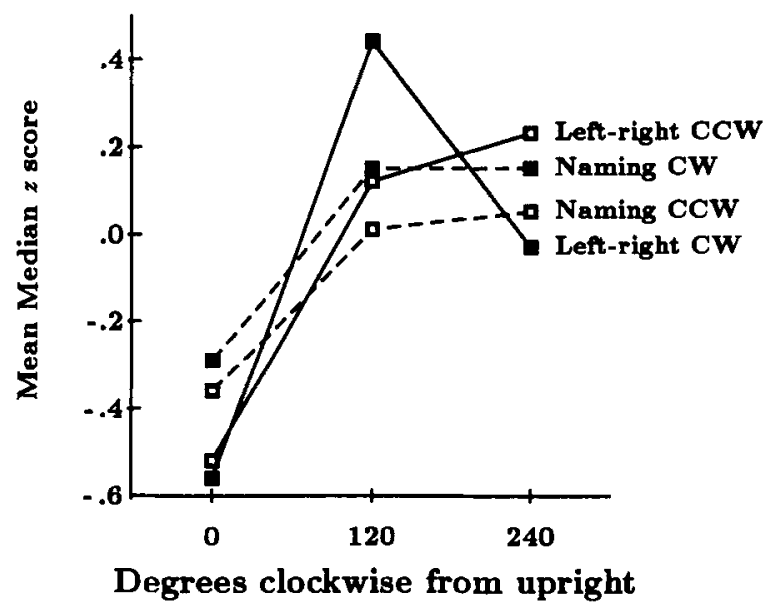

Figure 1. Mean median response time (RT) (in milliseconds; top panel) and accuracy (proportion correct, middle panel) in the subject analyses and mean median $z$ score of the RTs in the item analysis (bottom panel) in Experiment 1 in the naming and left-right tasks, as a function of the orientation of the objects and direction of the aftereffect (CW, clockwise; CCW, countenclockwise).

There were also significant effects of orientation $\left[F(2,88)=57.09, M S_{\mathrm{e}}=24,358.5, p<.0001\right]$, which are evident in Figure 1 (top). These effects were larger in the left-right task than in the naming task, however, which produced an interaction between task and orientation $\left[F(2,88)=4.41, M S_{\mathrm{e}}=24,358.5, p<.015\right]$. However, separate analyses showed that the orientation effects two tasks was also subjected to an ANOVA. The means are shown in the middle panel of Figure 1 . There was a significant main effect of orientation $\left[F(2,88)=10.71, M S_{\mathrm{e}}=\right.$ $0.007862, p<.0001]$, with accuracies of $.942, .888$, and .893 for the $0^{\circ}, 120^{\circ}$, and $240^{\circ}$ orientations, respectively. The interaction between orientation and the direction of the aftereffect approached significance $[F(2,88)=2.50$, $\left.M S_{\mathrm{e}}=0.007862, p<.088\right]$, but the three-way interaction between orientation, direction of the aftereffect, and task was not significant $\left[F(2,88)=0.93, M S_{\mathrm{e}}=0.007862\right.$, $p>$.39]. The interaction between orientation and the direction of the aftereffect was not significant in either task $\left[F(2,44)=1.82, M S_{\mathrm{e}}=0.007534, p>.17\right]$, for the leftright task; $F(2,44)=1.62, M S_{\mathrm{e}}=0.008189, p>.21$, for the naming task, in separate ANOVAs].

\section{Item Analyses}

We also performed analyses in which the observations were aggregated over items rather than over subjects. These analyses treat pictures as the random factor, instead of subjects, and so they permit generalization to the population of pictures of the same general type as those used in the experiment.

In order to reduce the effects of between-subjects variance (noise) from the item analyses, the RTs for correct trials for each subject were first converted to $z$ scores (based on each subject's mean RT and standard deviation for all correct trials). These $z$ scores were then aggregated over items, treating direction of aftereffect, orientation, and sides as within-items factors. Three items 
were excluded from the analyses because one or more cells had no valid observation, which left 69 items. As in the analysis of data aggregated over subjects, we computed the median score within each cell for each item and submitted the medians to an ANOVA. The means of these medians are shown in the bottom panel of Figure 1.

The pattern of results in the item analysis mirrored what we found in the subject analysis but with greater statistical sensitivity. The three-way interaction between task (left-right vs. naming), direction of aftereffect, and orientation (which was only marginally significant in the subject analysis) was clearly significant here $[F(2,136)=$ $\left.7.02, M S_{\mathrm{e}}=0.173417, p<.0015\right]$. The two-way interaction between orientation and direction of the aftereffect was also significant $\left[F(2,136)=9.37, M S_{\mathrm{e}}=\right.$ $0.187410, p<.0002]$. As expected, RTs were longer for larger orientations $\left[F(2,136)=101.93, M S_{\mathrm{e}}=0.303794\right.$, $p<.0001]$. The orientation effect was larger for the leftright task than for the naming task $[F(2,136)=12.26$, $\left.M S_{\mathrm{e}}=0.246151, p<.0001\right]$.

The effects of orientation were highly significant in both tasks $\left[F(2,136)=37.84, M S_{\mathrm{e}}=0.212032, p<.0001\right.$, for the naming task; $F(2,136)=76.82, M S_{\mathrm{e}}=0.337913$, $p<.0001$, for the left-right task]. This suggests that each task at least afforded the opportunity for the orientation effect to be modulated by the rotary aftereffect. However, the interaction between orientation and the direction of the aftereffect was significant only for the leftright task $\left[F(2,136)=15.69, M S_{\mathrm{e}}=0.186893, p<.0001\right]$. It did not approach significance for the naming task $\left[F(2,136)=0.24, M S_{\mathrm{e}}=0.173935, p>.79\right]$.

\section{Discussion}

For the left-right task, the RTs at orientations $120^{\circ}$ and $240^{\circ}$ were clearly influenced by the direction of the aftereffect. This was clear from the interaction between orientation and the direction of the aftereffect, although the effect was substantial only for the clockwise aftereffect. In contrast; although we observed substantial effects of orientation in the naming task, these effects did not vary with the direction of the rotary aftereffect. These results suggest that mental rotation was not responsible for the orientation effects observed in the naming task.

\section{EXPERIMENT 2}

Although the results of Experiment 1 were clear-cutthe rotary aftereffect interacted with orientation only in the left-right task and not in the naming task-one aspect of the results was less than perfect. The interaction between the direction of the rotary aftereffect and orientation in the left-right task was pronounced only for the clockwise aftereffect. The smaller size of the effect for the counterclockwise aftereffect was not expected.

In Experiment 2 we sought to extend the generality of the results obtained in Experiment 1. The logic of the design was the same. However, the method used to obtain an interaction with stimulus orientation was different. Rather than inducing a perceived rotation by means of an aftereffect, we rotated the objects physically during their presentation (see, e.g., Jolicoeur \& Cavanagh, 1992). We also increased the statistical power of the design by manipulating all factors in a within-subjects design.

\section{Method \\ Subjects}

The subjects were 48 undergraduates who participated voluntarily.

Stimuli

Eighty-four drawings of common objects from Snodgrass and Vanderwart (1980) were chosen. Each object was reflected about the vertical to produce a left-facing and a right-facing version for each object.

In each trial, an object was presented in eight different views formed by successive $2^{\circ}$ rotations. The orientation used to designate a trial was the orientation of the final view. Consider, for example, the $120^{\circ}$ counterclockwise condition. The presentation sequence for this condition was $134^{\circ}, 132^{\circ}, 130^{\circ}, 128^{\circ}, 126^{\circ}, 124^{\circ}, 122^{\circ}, 120^{\circ}$. This procedure ensures that any effect of the average orientation of the object during the display sequence will actually attenuate any interaction between orientation and direction of rotation. For example, in the $120^{\circ}$ counterclockwise condition, the mean orientation of the objects during the display sequence is $127^{\circ}$. The greater orientation should increase mean RT. Because the direction of rotation is counterclockwise, however, we expected a smaller RT for this condition than for the $120^{\circ}$ clockwise condition.

A blank screen followed the last image (i.e., there was no mask). Each view was displayed for $14 \mathrm{msec}$, with no intervening time between successive views, for a total presentation time of $112 \mathrm{msec}$. Given that the object rotated $2^{\circ}$ every $14 \mathrm{msec}$, the rate of rotation was about $143 \% \mathrm{sec}$.

\section{Design}

Each subject performed two blocks of 84 trials, one for each task. The order of tasks was counterbalanced across subjects, with an equal number of subjects assigned to each order. In each block, a different picture was shown on each trial. Twenty-eight pictures were shown in each of the three orientations $\left(0^{\circ}, 120^{\circ}\right.$, and $\left.240^{\circ}\right) ; 14$ were left-facing and 14 right-facing when upright, and 7 or each of these were shown rotating clockwise and 7 were shown rotating counterclockwise.

\section{Procedure}

The subject was asked to look directly at a fixation point at the middle of the screen. In each task, the subject initiated each trial by pressing the space bar, which triggered the stimulus presentation. The naming task was the same as in Experiment 1, except that accuracy was scored by the experimenter. The left-right task was also the same except for the response buttons (the $<$ key for the response left-facing and the $>$ key for the response right-facing). Each block was preceded by 20 practice trials, in which 10 objects not included in the experimental trials were each presented twice, each time in a different orientation.

There was a 1-sec intertrial interval. Each subject was tested with a new random order of the stimuli in each block of trials. Across all subjects, each object was presented equally often in each combination of the independent variables (orientation, left-right face direction, direction of rotation, and task). Each object was seen twice by each subject (once in each task).

\section{Results}

As in Experiment 1, two kinds of analyses were performed: one based on data aggregated over subjects and one based on data aggregated over items. An initial set of analyses of the results aggregated over subjects considered task order as a between-subjects variable. No interesting effects emerged from these analyses, so we performed subsequent analyses without this factor. 


\section{Subject Analyses}

Response times. The median RT for the correct responses in each cell of the design (task by orientation by side) was computed for each subject. The medians were then subjected to an ANOVA in which all factors were within-subjects. As in Experiment 1, the sides factor did interact with direction of rotation (only for the left-right task) but not with the joint effects of direction of rotation and orientation, which is the main focus of the empirical work, so this factor was not considered further. The means of the medians are shown in the top panel of Figure 2.

As in Experiment 1 naming RTs $(960 \mathrm{msec})$ were longer than left-right RTs $(774 \mathrm{msec})[F(1,47)=58.14$, $\left.M S_{\mathrm{e}}=85,514.0, p<.0001\right]$. RTs were affected by orientation $\left[F(2,94)=80.07, M S_{\mathrm{e}}=13,858.7, p<.0001\right]$. The effects of orientation were larger in the left-right task than in the naming task, which produced an interaction between task and orientation $\left[F(2,94)=19.28, M S_{\mathrm{e}}=\right.$ $10,878.1, p<.0001]$.

The critical question was whether the direction of object rotation would influence the orientation function. The interaction between these variables was highly significant $\left[F(2,94)=21.53, M S_{\mathrm{e}}=16,680.0, p<.0001\right]$, as was the triple interaction between task, direction of rotation, and orientation $\left[F(2,94)=18.21, M S_{\mathrm{e}}=12,072.8, p<.0001\right]$. Figure 2 (top) shows that the interaction between orientation and direction of rotation was clearly present for the left-right task but was negligible for the naming task.

Separate ANOVAs were also carried out for each task. The interaction between the direction of object rotation and orientation was significant for the left-right task $\left[F(2,94)=40.56, M S_{\mathrm{e}}=13,975.7, p<.0001\right]$ but not for the naming task $\left[F(2,94)=0.82, M S_{\mathrm{e}}=14,777.1, p>\right.$ .44]. These analyses also provide evidence that the lack of influence of the object rotation on the naming task was not due to high error variance in the naming task, because the $M S_{\mathrm{e}}$ estimates were similar across tasks.

We also performed analyses within each task, using only the results for the $120^{\circ}$ and $240^{\circ}$ conditions, where we expected the interaction between orientation and direction of rotation to be the strongest. A highly significant effect was found for the left-right task $[F(1,47)=$ $50.42, M S_{\mathrm{e}}=22,482.6, p<.0001$ ], but even this more sensitive test of the interaction was not significant for the naming task $\left[F(1,47)=0.95, M S_{\mathrm{e}}=17,838.4, p>.33\right]$.

Accuracy. The proportion of correct responses in the two tasks was also subjected to ANOVA with task, orientation, direction of rotation, and facing side as withinsubjects factors. Given that there were no interactions involving sides and direction of rotation, we performed subsequent analyses without the sides factor. The means for the remaining factors can be seen in the middle panel of Figure 2. Accuracy was higher, overall, in the leftright task $(.883)$ than in the naming task $(.812)[F(1,47)=$ $\left.21.03, M S_{\mathrm{e}}=0.034025, p<.0001\right]$. There was a significant main effect of orientation $\left[F(2,94)=28.14, M S_{\mathrm{e}}=\right.$ $0.011578, p<.0001]$. The interaction between orienta-
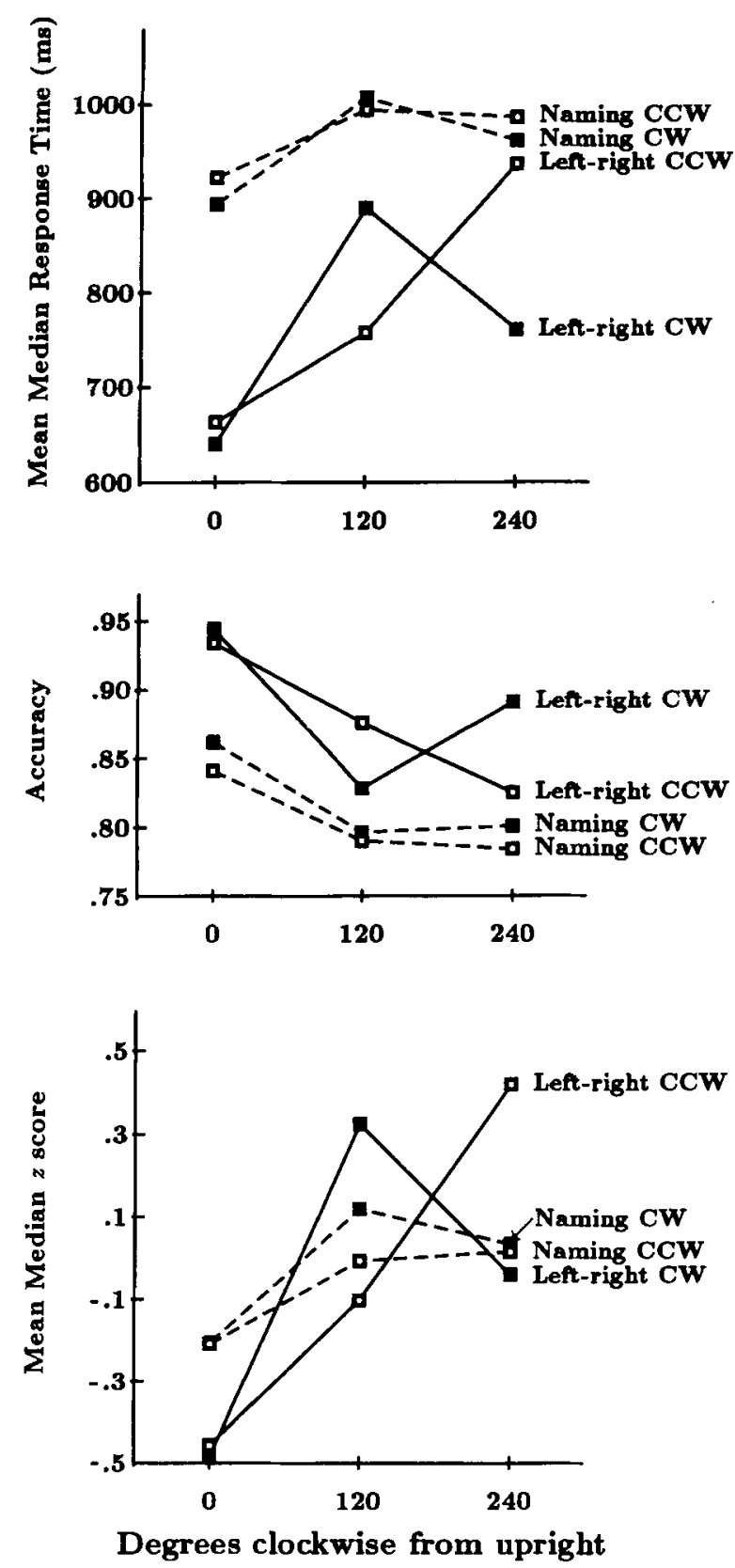

Figure 2. Mean median response time (RT) (in milliseconds; top panel) and accuracy (proportion correct, middle panel) in the subject analyses and mean median $z$ score of the RTs in the item analysis (bottom panel) in Experiment 2 in the naming and left-right tasks, as a function of the orientation of the objects and direction of object rotation (CW, clockwise; CCW, counterclockwise).

tion and the direction of object rotation was significant $\left[F(2,94)=5.41, M S_{\mathrm{e}}=0.008607, p<.006\right]$.

No other effect approached significance $(p>.18$ in all cases), except for the three-way interaction between task, direction of rotation, and orientation $[F(2,94)=3.34$, 
$\left.M S_{\mathrm{e}}=0.009624, p<.04\right]$. As is evident in the middle panel of Figure 2, the pattern of results mirrored those found in the RTs: Object rotation interacted with orientation for the left-right task but not for the naming task. Separate analyses for the two tasks corroborated this interpretation. The interaction between orientation and the direction of object rotation was highly significant for the left-right task $\left[F(2,94)=7.97, M S_{\mathrm{e}}=0.009703, p<\right.$ .0006] but not significant for the naming task $[F(2,94)=$ $\left.0.16, M S_{\mathrm{e}}=0.008528, p>.84\right]$.

\section{Item Analyses}

We used the same method of analysis as that used in Experiment 1 . One item was excluded from the analyses because one or more cells had no valid observation, which left 83 items. As in the analysis of data aggregated over subjects, we computed the median score within each cell for each item and submitted the medians to an ANOVA. The means of these medians can be seen in the bottom panel of Figure 2.

The pattern of results in the item analysis mirrored what we found in the subject analysis, but with even greater statistical sensitivity. The three-way interaction between task (left-right vs. naming), direction of rotation, and orientation was highly significant $[F(2,164)=$ $\left.23.42, M S_{\mathrm{e}}=0.134569, p<.0001\right]$. The two-way interaction between orientation and direction of object rotation was also significant $\left[F(2,164)=32.72, M S_{\mathrm{e}}=0.158033\right.$, $p<.0001]$. As expected, RTs were longer for larger orientations $\left[F(2,164)=122.69, M S_{\mathrm{e}}=0.170974, p<\right.$ $.0001]$, but this effect was larger for the left-right task than for the naming task $\left[F(2,164)=27.39, M S_{\mathrm{e}}=0.149403\right.$, $p<.0001]$.

Separate analyses for each task confirmed what is apparent by visually inspecting Figure 2 (bottom) and what was found in the analysis of data aggregated over subjects (Figure 2, top). There was no interaction between orientation and direction of rotation for the naming task $\left[F(2,164)=1.15, M S_{\mathrm{e}}=0.160571, p>.32\right]$. In contrast, this interaction was highly significant in the left-right task $\left[F(2,164)=61.64, M S_{\mathrm{e}}=0.132031, p<.0001\right]$.

\section{Discussion}

The results were clear-cut: A robust interaction between the direction of object rotation and orientation was found for the left-right task, whereas direction of object rotation had no apparent effect in the naming task. These results converge nicely with those of Experiment 1, in which a similar pattern of results was found when objects appeared to rotate as a result of a motion aftereffect.

\section{GENERAL DISCUSSION}

M. C. Corballis and McLaren (1982) investigated the joint effects of pattern orientation and of perceived rotary motion in a task that was believed to induce mental rotation (mirror image judgments on rotated alphanumeric characters). They induced a perception of rotary motion by means of a motion aftereffect that was produced by adapting to a rotating stimulus shown before each trial. They found that RTs at oblique orientations (e.g., $120^{\circ}$ or $240^{\circ}$ ) were affected by the direction of per- ceived rotation of the stimulus. RTs were significantly elevated when the direction of perceived rotation was in the direction opposite to the shortest path of mental rotation, but they were not facilitated when the aftereffect was in the same direction as the path of mental rotation. Jolicoeur and Cavanagh (1992) found both facilitation and inhibition when the perceived rotation was produced by an actual rotating physical stimulus, but otherwise the pattern of results was very similar to that of M. C. Corballis and McLaren (1982).

There are several hypotheses concerning the exact nature of the interaction between perceived rotary motion and orientation in tasks that induce mental rotation. The three most plausible (not mutually exclusive) accounts are: (1) the rotary motion could alter the perceived orientation of the stimulus; (2) it could influence the rate of mental rotation; and (3) it may induce the observer to rotate through the larger $\left(240^{\circ}\right)$ angle to the upright.

The results of M. C. Corballis and McLaren (1982) were weakly supportive of the third hypothesis (see also M. C. Corballis, 1986). However, this account would not explain how a congruent physical rotation could produce facilitation (Jolicoeur \& Cavanagh, 1992), unless subjects rotate through the larger angle in the neutral condition (something that is not supported by the reaction-time distribution analyses of Cooper \& Shepard, 1973). Recent work by Heil, Bajrić, Rösler, and Hennighausen (1997) suggests that it is likely that both the rate of rotation and the direction of rotation are affected by the rotary aftereffect.

The absence of interactions between perceived rotation and orientation in the naming task provides evidence against the first hypothesis. If the perceived orientation had been altered by the experimental manipulations, then naming times should have been affected, because we know that naming time does depend on orientation. Thus, the fact that naming time was not affected suggests that perceived orientation was not influenced strongly either by the rotary aftereffect or by the actual rotation of the stimulus.

For present purposes, however, what is most critical is the assumption that the interaction between orientation and direction of perceived rotary motion can be found whenever mental rotation is the underlying mechanism producing an observed effect of orientation. If so, the fact that there was no observable modulation of orientation effects by perceived rotary motion in the naming task can be taken as evidence that mental rotation was not involved.

M. C. Corballis (1988) suggested that observers in naming tasks use a mental-rotation strategy only intermittently, perhaps as an occasional check on an earlier but cruder identification process. Jolicoeur (1990), on the other hand, suggested that mental rotation could provide the basis for identification for orientation up to $120^{\circ}$, but that a featurebased system sometimes achieves the answer first, and that the likelihood that the mental rotation process would achieve identification first depended on orientation (more likely for smaller orientations) and on practice (less likely with more practice). Had mental rotation been used on a substantial proportion of trials (e.g., proportional to the slope difference across tasks), however, one would have expected the aftereffect to have influenced naming times to a proportional extent. The resuits did not support this possibility and suggest that both of these proposals are incorrect. The most straightforward interpretation of the present results is that the orientation effects observed when familiar objects are identified are not the result of mental rotation. Some other mechanism-such as the frame-selection relaxation process proposed by Hinton (1981), the image interpolation process suggested by Bülthoff and Edelman (1992), or some normalization process other than mental rotation - probably mediates the recognition of rotated objects.

\section{REFERENCES}

Bülthoff, H. H., \& Edelman, S. (1992). Psychophysical support for a two-dimensional view interpolation theory of object recognition. Proceedings of the National Academy of Sciences, 89, 60-64.

COOPER, L. A., \& Shepard, R. N. (1973). Chronometric studies of the rotation of mental images. In W. G. Chase (Ed.), Visual information processing (pp. 75-176). New York: Academic Press.

Corballis, M. C. (1982). Mental rotation: Anatomy of a paradigm. In 
M. Potegal (Ed.), Spatial abilities: Developmental and physiological foundations (pp. 173-198). New York: Academic Press.

Corballis, M. C. (1986), On imagined revolutions. In D. F. Marks (Ed.), Theories of image formation (pp. 151-168). New York: Brandon House.

Corballis, M. C. (1988). Recognition of disoriented shapes. Psychological Review, 95, 115-123.

Corballis, M. C., \& Blackman, A. R. (1990). The effect of apparent movement on mental rotation. Memory \& Cognition, 18, 551-555.

Corballis, M. C., \& McLaren, R. (1982). Interaction between perceived and imagined rotation. Journal of Experimental Psychology: Human Perception \& Performance, 8, 215-224.

Corballis, P. M., \& Corballis, M. C. (1993). The effect of apparent motion on mental rotation: Push or pull? Memory \& Cognition, 21 458-466.

Heil, M., Bajrić, J., Rösler, F., \& Hennighausen, E. (1997). A rotation aftereffect changes both the speed and the preferred direction of mental rotation. Journal of Experimental Psychology: Human Perception \& Performance, 23, 681-692.

Hinton, G. E. (1981). A parallel computation that assigns canonical object-based frames of reference. In Proceedings of the Seventh International Joint Conference on Artificial Intelligence (Vol. 2, pp. 683685). Los Altos, CA: Kaufman.

Jolicoevr, P. (1985). The time to name disoriented natural objects. Memory \& Cognition, 13, 289-303.

JoliCOEUR, P. (1988). Mental rotation and the identification of disoriented objects. Canadian Journal of Psychology, 42, 461-478.

JOLICOEUR, P. (1990). Identification of disoriented objects: A dualsystems theory. Mind \& Language, 5, 387-410.
Jolicoeur, P., \& Cavanagh, P. (1992). Mental rotation, physical rotation, and input channels in vision. Journal of Experimental Psychology: Human Perception \& Performance, 18, 371-384.

JolicoevR, P., \& MillikeN, B. (1989). Identification of disoriented objects: Effects of context of prior presentation. Journal of Experimental Psychology: Learning, Memory, \& Cognition, 15, 200-210.

MAKI, R. H. (1986). Naming and locating the tops of rotated pictures. Canadian Journal of Psychology, 40, 368-387.

MURRAY, J. E. (1995). Imagining and naming rotated natural objects. Psychonomic Bulletin \& Review, 2, 239-243.

Murray, J. E., Jolicoeur, P., McMullen, P. A., \& Ingleton, M. (1993). Orientation invariant transfer of training in the identification of rotated natural objects. Memory \& Cognition, 21, 604-610.

ShePARD, R. N., \& COOPER, L. A. (1982). Mental images and their transformations. Cambridge, MA: MIT Press.

SHWARTZ, S. P. (1981). The perception of disoriented complex objects. Unpublished manuscript, Yale University, New Haven, CT.

SNODGRASS, J. G., \& VANDERWART, M. (1980). A standardized set of 260 pictures: Norms for name agreement, image agreement, familiarity, and visual complexity. Journal of Experimental Psychology: Human Learning \& Memory, 6, 174-215.

TARR, M. J., \& PINKER, S. (1989). Mental rotation and orientationdependence in shape recognition. Cognitive Psychology, 21, 233282.

(Manuscript received March 31, 1996; revision accepted for publication October 7, 1997.) 